\title{
A systematic review and meta-analysis study of salivary and serum interleukin-8 levels in oral lichen planus
}

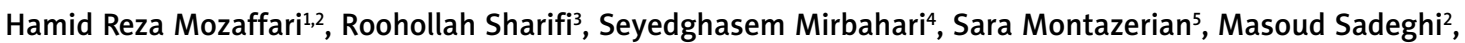 \\ Sedigheh Rostami
}

${ }^{1}$ Department of Oral and Maxillofacial Medicine, School of Dentistry, Kermanshah University of Medical Sciences, Kermanshah, Iran ${ }^{2}$ Medical Biology Research Center, Kermanshah University of Medical Sciences, Kermanshah, Iran

${ }^{3}$ Department of Endodontics, School of Dentistry, Kermanshah University of Medical Sciences, Kermanshah, Iran ${ }^{4}$ Department of Pathology, School of Medicine, Kermanshah University of Medical Sciences, Kermanshah, Iran ${ }^{5}$ Students Research Committee, Kermanshah University of Medical Sciences, Kermanshah, Iran

Adv Dermatol Allergol 2018; XXXV (6): 599-604 DOI: https://doi.org/10.5114/ada.2018.77611

\begin{abstract}
Introduction: Interleukin-8 (IL-8) is an important mediator of the host response to injury and inflammation that the clinical severity of oral lichen planus (OLP) is associated with elevated serum IL-8 levels.

Aim: To evaluate serum and salivary IL-8 levels of the OLP patients compared with the healthy controls and measurement of IL-8 level in which sample is more valuable.

Material and methods: Five databases including PubMed/Medline, Web of Science, Science Direct, Cochrane Library and Scopus were searched for the evaluation of serum and salivary IL-8 levels of the OLP patients compared with the healthy controls in the English abstract. The Newcastle-Ottawa Quality Assessment Scale (NOS) was used for checking the quality of the studies. A random-effect model was used for calculating the mean difference (MD) and 95\% confidence intervals (Cls).

Results: Nine studies were included in the meta-analysis. The pooled estimate showed a significant difference between two groups that the salivary IL-8 level in the OLP patients was higher than the healthy controls (MD = 766.32 pg/ $\mathrm{ml}, 95 \% \mathrm{Cl}: 394.90-1137.75 ; p<0.0001)$ and also the serum IL-8 level in the OLP patients was higher than the healthy controls ( $\mathrm{MD}=8.38 \mathrm{pg} / \mathrm{ml}, 95 \% \mathrm{Cl}: 3.32-13.44 ; p=0.001)$.

Conclusions: The higher levels of IL-8 in saliva compared with serum suggest that measurement of this marker in saliva may be more useful than serum measurements for determining therapeutic and diagnostic aims.
\end{abstract}

Key words: oral lichen planus, interleukin-8, saliva, serum.

\section{Introduction}

Oral lichen planus (OLP) is an autoimmune and chronic inflammatory disease affecting the oral mucosa [1] and may also involve the skin and the genitalia [2]. Its incidence is higher in women than in men with a different age range around the world [3]; it may be divided clinically as a reticular, erythematous or erosive type of lesions [4]. This is associated with various other systemic conditions such as hypertension or diabetes mellitus [5]. The exact etiology of OLP is unclear, but the immunological system plays a significant role [6]. The investigations have demonstrated that immune activation and chronic inflammation may be one of the causes of oncogenesis [7]. Oral lichen planus has been documented, in a small percentage of cases (1-3\%), to undergo malignant transformation [8, 9]. The nuclear factor- $\kappa \mathrm{B}(\mathrm{NF}-\mathrm{\kappa} \mathrm{B})$ is a primary transcription factor controlling the expression of a series of cytokines with proinflammatory, pro-angiogenic, and immunoregulatory activity, including TNF- $\alpha$, interleukin-1 $\alpha$, interleukin-6 (IL-6), and interleukin-8 (IL-8) [10]. Interleukin-8 is an important mediator of the host response to injury and inflammation [11] and has a primary role in chronic diseases [12]. It possesses diverse functions as a neutrophil activator and a chemoattractant for neutrophils, T cells, and basophils [13]. Because the clinical severity of OLP is associated with elevated serum IL-8 levels [9], IL-8 serves as a reliable tool to assess the clinical severity of this disease. Evidently it is important to evaluate the practical application of these salivary analyses of NF- $\mathrm{B}$-dependent cytokines in the clinical management of OLP [14].

Address for correspondence: Masoud Sadeghi, Medical Biology Research Center, Kermanshah University of Medical Sciences, 67145-1673 Kermanshah, Iran, phone: +989185960644, e-mail: sadeghi_mbrc@yahoo.com Received: 23.08.2017, accepted: 10.10.2017. 


\section{Aim}

The aim of this meta-analysis was to evaluate serum and salivary IL-8 levels of the OLP patients compared with the healthy controls and measurement of IL-8 level in which sample is more valuable.

\section{Material and methods}

\section{Search strategies}

Five databases (PubMed/Medline, Web of Science, Science Direct, Cochrane Library and Scopus) were searched from 1983 to March 2017 for English-language publications using the key words "oral lichen planus" or "OLP" combined with "interleukin-8" or "IL-8".

\section{Study selection}

One author (M.S.) searched the articles and then the second author (H.R.M.) blinded to the first reviewer, if there was any disagreement between two authors, the third author (R.S) resolved the problem. The forth author (S.M.) did the final revision. The studies were searched for the evaluation of serum and salivary IL-8 levels of the OLP patients compared with the healthy controls in the English abstract. Studies were included if: a) they had the OLP and healthy control groups, b) they evaluated serum or salivary IL-8 levels of the OLP patients, $c$ ) they diagnosed OLP based on clinical, histological methods or both by the World Health Organization (WHO) [15], and d) the controls did not have OLP and any other cutaneous dermatological or systematic disease and also the OLP patients did not have any other dermatological or systematic disease.

\section{Data extraction}

Name of the author, year of publication, country of the region, number of the OLP patients, number of the control subjects, male/female for both groups, the age for both groups (mean \pm SD), levels of IL-8 (mean \pm SD) were the relevant data extracted for every study. Serum and salivary IL-8 levels were measured with the immunoenzymatic technique (ELISA) performed with a human IL-8 high-sensitivity ELISA kit. The unit of measurement used across studies was $\mathrm{pg} / \mathrm{ml}$. If the data were presented using the standard error (SE), then the formula of $\mathrm{SE}=\frac{\mathrm{SD}}{\sqrt{N}}$ was used to calculate SD ( $N=$ sample size).

\section{Quality evaluation}

One reviewer (M.S) evaluated the quality of each included study using the Newcastle-Ottawa Quality Assessment Scale (NOS) to determine the quality of selection of study participants, comparability, exposure and outcome, with a maximum score of 9 points. The studies quality was divided into three categories: high quality was scored 7 to 9 , moderate quality scored 4 to 6 , and low quality scored 0 to $3[16]$.

\section{Statistical analysis}

The data were analyzed (a random-effect model) with Review Manager 5.3 (RevMan 5.3, The Cochrane Collaboration, Oxford, United Kingdom) using mean difference (MD) and 95\% confidence intervals (Cls). The MD of the studies was calculated in comparison to the estimate of serum or salivary IL-8 levels of the OLP patients compared with the control subjects by meta-analysis. The $Q$ and $I^{2}$ statistics were used for evaluation of heterogeneity between estimates that for the $\mathrm{Q}$ statistics, heterogeneity was considered as $p<0.1$. $P$-value ( 2 -sided) $<0.05$ was considered to be statistically significant in the analyses. The publication bias was assessed through funnel plot analysis with the Begg's and Egger's tests.

\section{Results}

\section{Study selection}

Out of 112 studies searched in databases and after duplications removed, 63 studies were screened; 37 studies were not relevant and therefore excluded. Twenty-six studies were evaluated based on a full-text basis; 17 studies excluded for some reasons (Figure 1). At last, 9 studies were included and analyzed for meta-analysis study.

\section{Characteristics of the studies}

Nine studies included in meta-analysis were reported from 2005 to 2016 (Table 1). Four studies reported salivary IL-8 levels [7, 8, 14, 17], three studies serum IL-8 levels $[9,18,19]$, and two studies both salivary and serum IL-8 levels [20, 21]. Four studies reported the participants from the USA [7, 8, 14, 17], one from Taiwan [9], two from China [18, 20], one from Belgium [21], and one from Iran [19]. All studies included 536 OLP patients and 316 healthy controls. One study [18] censored eight patients in the final analysis.

\section{Meta-analysis study}

The studies on saliva (6 studies) included 144 OLP patients and 140 healthy controls (Figure 2). The pooled estimate showed a significant difference between two groups as the salivary IL-8 level was higher in the OLP patients than the healthy controls ( $M D=766.32 \mathrm{pg} / \mathrm{ml}, 95 \% \mathrm{Cl}$ : 394.90-1137.75; $p<0.0001)$. The studies on serum ( 5 studies) included 414 OLP patients and 256 healthy controls. The pooled estimate showed a significant difference between two groups; the serum IL-8 level was higher in the OLP patients than the healthy controls (MD $=8.38 \mathrm{pg} / \mathrm{ml}$, 95\% Cl: 3.32-13.44; $p=0.001$ ). Also, the IL-8 level was significantly higher in saliva compared with serum $(p<0.0001)$ 
(around 91 times). Heterogeneity between salivary studies was $94 \%$ and for serum studies was $98 \%$.

\section{Quality evaluation}

The quality score for each study in meta-analysis has been shown in Table 2. The mean quality score of nine studies was 6.78 (moderate quality).

\section{Publication bias}

Figure 3 shows the funnel plot of the studies in meta-analysis. Begg's and Egger's tests did not reveal any significant evidence of publication bias among the included studies on saliva (Begg's test, $p=0.347$; Egger's test, $p=0.108$ ) (Figure $3 \mathrm{~A}$ ) and also among the included studies on serum (Begg's test, $p=0.624$; Egger's test, $p=0.492$ ) (Figure $3 \mathrm{~B}$ ).

\section{Discussion}

The immunological system such as cytokines plays a significant role in pathogenesis and progression of OLP [5]. Therefore, this study evaluated the levels of IL-8 as a proinflammatory cytokine in immune and inflammatory responses. A random-effect model was done to calculate the pooled estimates because there was a significant heterogeneity between studies. The pooled estimate showed that salivary and serum IL-8 level of the OLP patients was significantly superior to the healthy controls. The results of Begg's and Egger's tests showed the lack of significant publication bias. The meta-analysis study included six studies on saliva [7, 8, 14, 17, 20, 21] and five studies on serum [9, 18-21]; each study reported salivary and serum IL-8 levels of the OLP patients to be significantly superior to the healthy controls. Also, IL-8 level in saliva was superior (around 91 times) to serum and the meta-analysis suggests measurement of a salivary level of this marker is better than the serum level for treatment aims. Most studies of meta-analysis matched the OLP patients and the healthy controls in terms of age and sex.

Oral lichen planus is a T cell-mediated chronic inflammatory disorder which has been considered an autoimmune disease affecting the oral mucosa [22]. It has also been reported that the clinical severity of OLP is directly associated with the IL-8 level in the serum [9]. The high levels of IL-8 had a correlation with an increased risk of development of cancers and warrant much closer followups [23]. Administration of medications balancing the IL-8 level and decreasing its serum level may be a step forward to alleviate the symptoms of OLP [19].

One study indicated that analysis of NF- $\mathrm{B} B$-dependent cytokines in whole unstimulated saliva could be one of predictive means to reflect the trend of malignant transformation of OLP [7]. As oral fluid analysis has some obvious advantages compared with blood-based analy-

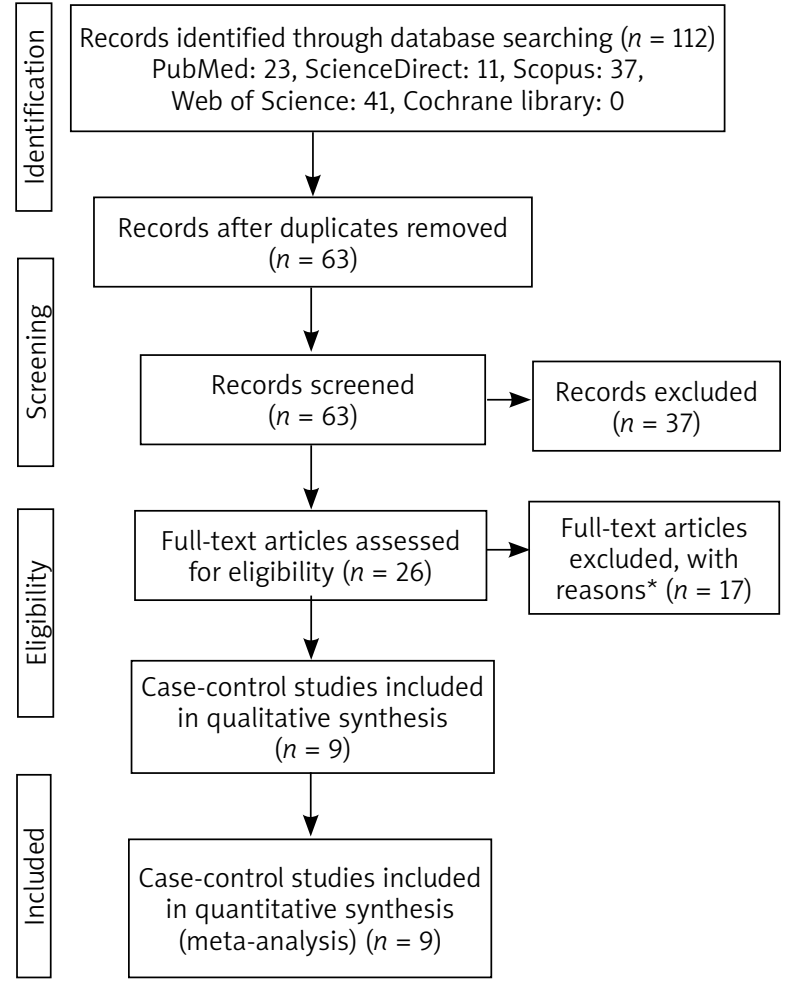

*1 reported IL-8 polymorphism; 2 were conference paper; 1 was trial; 1 was short communication; 1 was review study; 4 didn't have healthy control; 1 was mini-review; 1 didn't report mean IL-8 in baseline; 1 reported types of oral premalignant lesions in a group; 2 were meeting abstract; 1 reported mean IL- 8 in lesion tissue transudates; 1 didn't report mean IL-8

Figure 1. Flowchart of the study

ses, such as easy/safe access and non-invasive collection, oral fluids have been widely used in drug and disease monitoring and the detection of various oral and systemic maladies [24, 25]. Therefore, the detection of NF$\kappa \mathrm{B}$-dependent cytokines in OLP is likely to have clinical potential for monitoring disease activity and therapeutic response of OLP [14]. Salivary IL-8 levels in patients with OLP are significantly decreased after dexamethasone treatment [14], which indicates that the levels of this salivary protein could be influenced by the degree of inflammation in OLP. The results indicated that the absolute concentrations of cytokines/chemokines such as IL-8 in oral fluids can be fully detectable, and this cytokine can be used as an alternative biomarker to blood and other body fluids. [1] Therefore, cytokine and anticytokine therapies have shown some potential in the treatment of autoimmune diseases such as OLP [8].

Salivary IL-8 is a more sensitive marker for monitoring the disease activity and therapeutic responses in OLP so its detection may be used to more precisely describe the activity of OLP compared with serum [20]. Thus, IL-8 
Table 1. Characteristics of the studies included in meta-analysis $(n=9)$

\begin{tabular}{|c|c|c|c|c|c|c|}
\hline $\begin{array}{l}\text { The first author of study } \\
\text { [year] }\end{array}$ & Country & $\begin{array}{l}\text { Number of } \\
\text { OLP patients }\end{array}$ & $\begin{array}{l}\text { Number of } \\
\text { controls }\end{array}$ & $\begin{array}{l}\text { Age (mean), years/ } \\
F: M \text { of OLP patients }\end{array}$ & $\begin{array}{l}\text { Age (mean), years/ } \\
\mathrm{F}: \mathrm{M} \text { of controls }\end{array}$ & Sample \\
\hline Rhodus (2005a) & USA & 13 & 13 & $57 / 13: 0$ & $58 / 13: 0$ & Saliva \\
\hline Rhodus (2005b) & USA & 13 & 13 & $57 / 9: 4$ & $59 / 3: 10$ & Saliva \\
\hline Sun (2005) & Taiwan & 158 & 54 & $51.3 / 43: 115$ & $-/ 24: 30$ & Serum \\
\hline Rhodus (2006) & USA & 13 & 13 & $57.2 / 0: 13$ & Matched/Matched & Saliva \\
\hline Zhang (2008) & China & 30 & 30 & $45 / 16: 14$ & $40 / 15: 15$ & $\begin{array}{l}\text { Saliva and } \\
\text { serum }\end{array}$ \\
\hline Lisa Cheng (2014) & USA & 21 & 21 & $61.8 / 4: 17$ & $62.9 / 9: 12$ & Saliva \\
\hline Zhu (2014) & China & 150 & 102 & $-/ 44: 106$ & $-/ 41: 61$ & Serum \\
\hline Kaur (2015) & Belgium & 54 & 50 & Range, 41.65/- & Range, 42-65/- & $\begin{array}{l}\text { Saliva and } \\
\text { serum }\end{array}$ \\
\hline Tavangar (2016) & Iran & 30 & 20 & $-/-$ & $-1-$ & Serum \\
\hline
\end{tabular}

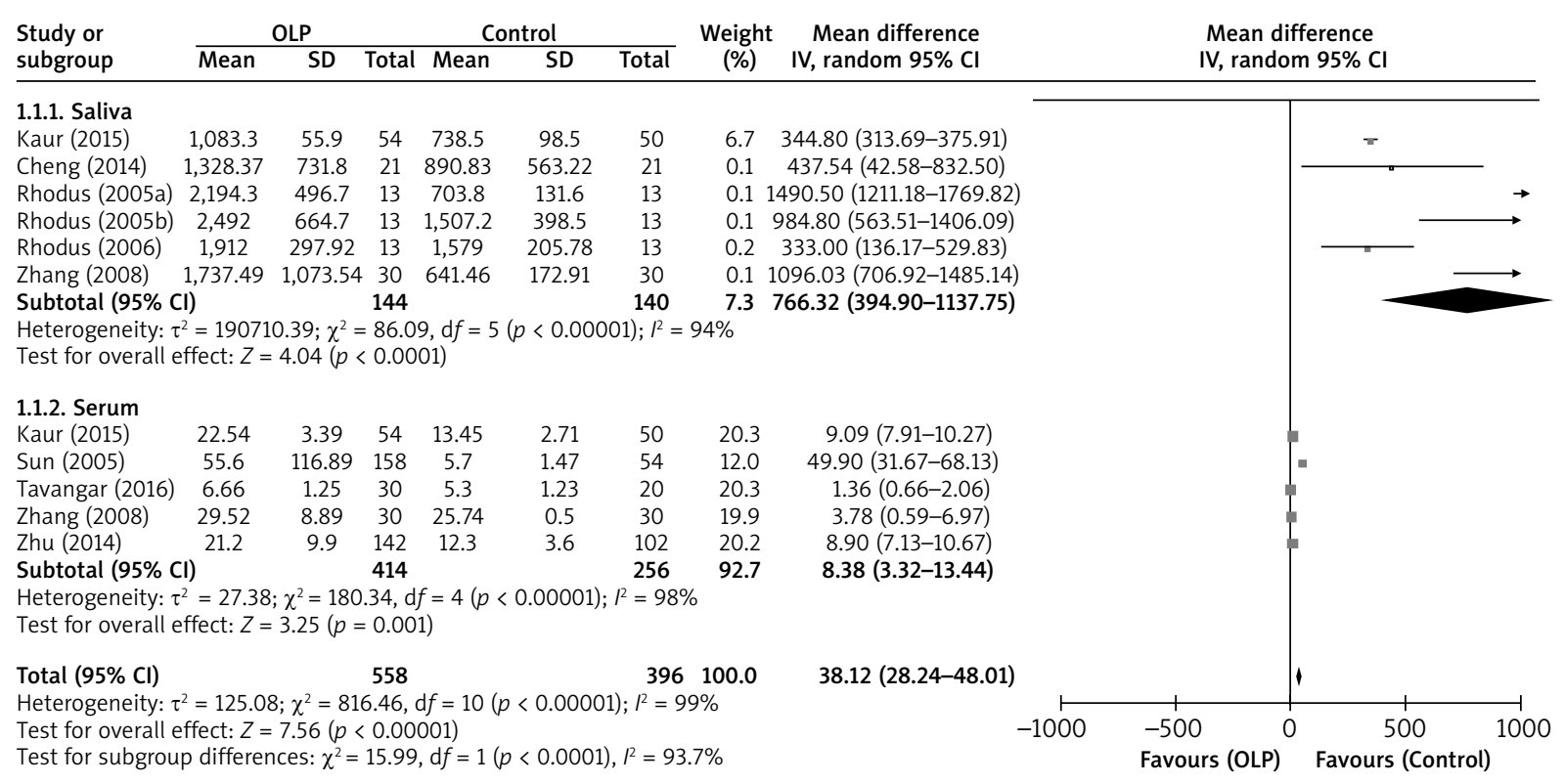

Figure 2. Forest plot of random-effect of interleukin-8 levels of saliva and serum in oral lichen planus groups compared with healthy controls

should be considered as an effective inflammatory factor in the pathogenesis of OLP. On the whole, the result indicates that the profile of some pro-inflammatory NF$\kappa \mathrm{B}$-dependent cytokines, TNF- $\alpha$ [6], IL-6, and IL-8, can be elevated to various degrees in both serum and salivary samples with OLP.

Also, the results suggested that IL-8 seems to play a key role in the transformation of reticular to erosive form, possibly by impairing the efficacy of repair mechanisms of keratinocyte damage [7]. Alikhani et al. [23] concluded that IL-8 levels in erosive form were more than on-erosive form. In a cohort study, the study suggested that the IL-8 polymorphisms may be associated with the severity of OLP and that the frequencies of IL-8 gene polymorphisms were different in two forms of OLP [26]. Despite age and gender matching in the studies of meta-analysis, the type of OLP and genetics can be other influential factors on IL-8 levels of OLP patients.

\section{Conclusions}

There were some limitations such as variable timing of saliva collection, different kits and methods of IL-8 assays, variation in criteria for selection of the healthy controls, varying type and severity of OLP across studies, lack of uniform matching of age and sex between the OLP patients and the healthy controls, and need to estimate a mean value and SD in several studies. However, 
Table 2. Quality ratings for the studies included on the basis of Newcastle-Ottawa quality assessment scale $(n=9)$

\begin{tabular}{lcccc}
\hline The first author of study [year] & Selection (score) & Comparability (score) & Exposure/outcome (score) & Total score \\
\hline Rhodus (2005a) & 3 & 1 & 3 & 7 \\
\hline Rhodus (2005b) & 3 & 2 & 3 & 8 \\
\hline Sun (2005) & 3 & 1 & 3 & 7 \\
\hline Rhodus (2006) & 2 & 1 & 3 & 6 \\
\hline Zhang (2008) & 3 & 2 & 2 & 7 \\
\hline Lisa Cheng (2014) & 3 & 2 & 2 & 7 \\
\hline Zhu (2014) & 2 & 2 & 3 & 6 \\
\hline Kaur (2015) & 3 & 2 & 2 & 5 \\
\hline Tavangar (2016) & 3 & 0 & 2.56 & 6.78 \\
\hline Mean scores & 2.78 & 1.44 & & 8 \\
\hline
\end{tabular}

A

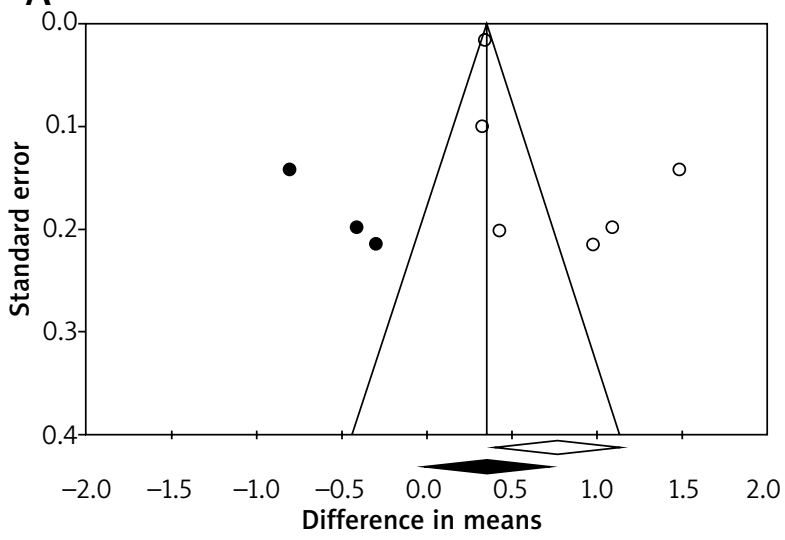

B

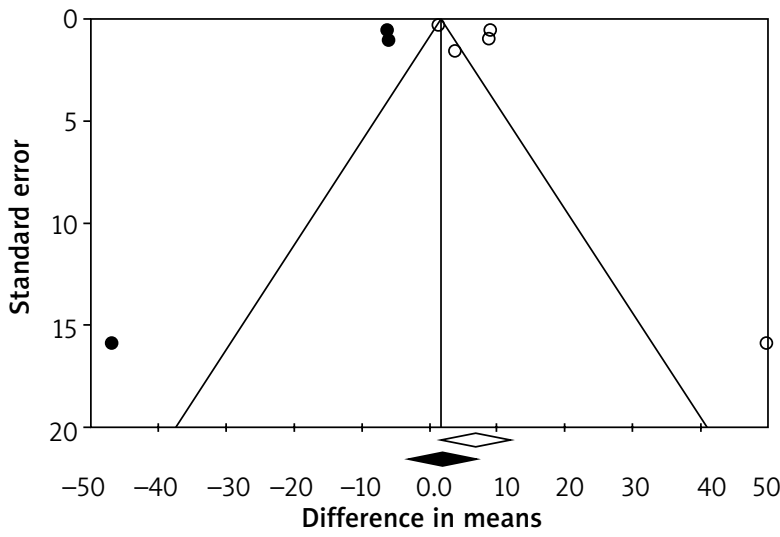

Figure 3. Funnel plot of random-effect of interleukin-8 of (A) saliva studies (mean $\times 10^{3}$ ) and (B) serum studies in oral lichen planus patients compared with healthy controls

the meta-analysis showed that the higher levels of IL-8 in saliva compared with serum suggest that measurement of this marker in saliva may be more useful than serum measurements for determining therapeutic and diagnostic aims. Other confounding factors such as the type of OLP and genetics must be taken in interpreting results.

\section{Acknowledgments}

The authors gratefully acknowledge the Research Council of Kermanshah University of Medical Sciences (Grant Number: 96171) for the financial support. This work was performed in partial fulfillment of the requirements for (General Dentistry) of (Sara Montazerian), in Faculty of Medicine, Kermanshah University of Medical Sciences, Kermanshah, Iran.

\section{Conflict of interest}

The authors declare no conflict of interest.

\section{References}

1. Drogoszewska B, Chomik P, Polcyn A, Michcik A. Clinical diagnosis of oral erosive lichen planus by direct oral microscopy. Postep Dermatol Alergol 2014; 31: 222-8.

2. Eisen D, Carrozzo M, Bagan Sebastian JV, Thongprasom K. Oral lichen planus: clinical features and management. Oral Dis 2005; 11: 338-49.

3. Mozaffari HR, Rahmani M, Rezaei F, et al. Evaluation of oral lichen planus frequency in patients referred to pathology centers of Kermanshah city, during 2008 to 2011. Sch J App Med Sci 2016; 4: 2200-2.

4. Lodi G, Scully C, Carrozzo M, et al. Current controversies in oral lichen planus: report of an international consensus meeting. Part 1. Viral infections and etiopathogenesis. Oral Surg Oral Med Oral Pathol Oral Radiol Endod 2005; 100: 40-51.

5. Mozaffari HR, Sharifi R, Sadeghi M. Prevalence of oral lichen planus in diabetes mellitus: a meta-analysis study. Acta Inform Med 2016; 24: 390-3.

6. Mozaffari HR, Ramezani M, Mahmoudiahmadabadi M, et al. Salivary and serum levels of tumor necrosis factor-alpha in oral lichen planus: a systematic review and meta-analysis study. Oral Surg Oral Med Oral Pathol Oral Radiol 2017; 124 : e183-9. 
7. Rhodus NL, Cheng B, Myers S, et al. The feasibility of monitoring NF-kappaB associated cytokines: TNF-alpha, IL-1alpha, IL-6, and IL-8 in whole saliva for the malignant transformation of oral lichen planus. Mol Carcinog 2005a; 44: 77-82.

8. Rhodus NL, Cheng B, Myers S, et al. A comparison of the pro-inflammatory, NF-kappaB-dependent cytokines: TNFalpha, IL-1-alpha, IL-6, and IL-8 in different oral fluids from oral lichen planus patients. Clin Immunol 2005b; 114: 278-83.

9. Sun A, Wang JT, Chia JS, Chiang CP. Serum interleukin-8 level is a more sensitive marker than serum interleukin- 6 level in monitoring the disease activity of oral lichen planus. $\mathrm{Br} J$ Dermatol 2005; 152: 1187-92.

10. Chen F, Castranova V, Shi X. New insights into the role of nuclear factor-kappaB in cell growth regulation. Am J Pathol 2001; 159: 387-97.

11. al-Dalaan A, al-Sedairy S, al-Balaa S, et al. Enhanced interleukin 8 secretion in circulation of patients with Behcet's disease. J Rheumatol 1995; 22: 904-7.

12. Shahriary A, Panahi Y, Shirali S, Rahmani H. Relationship of serum levels of interleukin 6, interleukin 8, and C-reactive protein with forced expiratory volume in first second in patients with mustard lung and chronic obstructive pulmonary diseases: systematic review and meta-analysis. Postep Dermatol Alergol 2017; 34: 192-8.

13. Wang LM, Kitteringham N, Mineshita S, et al. The demonstration of serum interleukin-8 and superoxide dismutase in Adamantiades-Behcet's disease. Arch Dermatol Res 1997; 289: 444-7.

14. Rhodus NL, Cheng B, Bowles W, et al. Proinflammatory cytokine levels in saliva before and after treatment of (erosive) oral lichen planus with dexamethasone. Oral Dis 2006; 12: 112-6.

15. Yamamoto T, Yoneda K, Ueta E, Osaki T. Serum cytokines, interleukin-2 receptor, and soluble intercellular adhesion molecule-1 in oral disorders. Oral Surg Oral Med Oral Pathol 1994; 78: 727-35.

16. Wells GA, Shea B, O'Connell D, et al. The Newcastle-Ottawa Scale (NOS) for assessing the quality of nonrandomised studies in meta-analyses. 2008. http://www.ohri. ca/programs/clinical_epidemiology/oxford.asp (accessed 1/03/2016).

17. Lisa Cheng YS, Jordan L, Gorugantula LM, et al. Salivary interleukin- 6 and -8 in patients with oral cancer and patients with chronic oral inflammatory diseases. J Periodontol 2014; 85: 956-65.

18. Zhu Y, Li J, Bai Y, et al. Hydroxychloroquine decreases the upregulated frequencies of Tregs in patients with oral lichen planus. Clin Oral Investig 2014; 18: 1903-11.

19. Tavangar A, Khozeimeh F, Ghoreishian F, Boroujeni MA. Serum level of Interleukin-8 in subjects with diabetes, diabetes plus oral lichen planus, and oral lichen planus: a biochemical study. Dent Res I (Isfahan) 2016; 13: 413-8.

20. Zhang Y, Lin M, Zhang S, et al. NF-kappaB-dependent cytokines in saliva and serum from patients with oral lichen planus: a study in an ethnic Chinese population. Cytokine 2008; 41: 144-9.

21. Kaur J, Jacobs R. Proinflammatory cytokine levels in oral lichen planus, oral leukoplakia, and oral submucous fibrosis. J Korean Assoc Oral Maxillofac Surg 2015; 41: 171-5.

22. Mignogna MD, Fedele S, Lo Russo L, et al. Immune activation and chronic inflammation as the cause of malignancy in oral lichen planus: is there any evidence? Oral Oncol 2004; 40: 120-30.
23. Alikhani M, Ghalaiani P, Askariyan E, et al. Association between the clinical severity of oral lichen planus and anti-TPO level in thyroid patients. Braz Oral Res 2017; 31: e10.

24. Kaufman E, Lamster IB. The diagnostic applications of saliva - a review. Crit Rev Oral Biol Med 2002; 13: 197-212.

25. Lawrence HP. Salivary markers of systemic disease: noninvasive diagnosis of disease and monitoring of general health. J Can Dent Assoc 2002; 68: 170-4.

26. Dan H, Liu W, Zhou Y, et al. Association of interleukin-8 gene polymorphisms and haplotypes with oral lichen planus in a Chinese population. Inflammation 2010; 33: 76-81. 